\title{
TOXICIDADE DE PESTICIDAS PARA ADULTOS DE ORIUS INSIDIOSUS (SAY, 1832) (HEMIPTERA: ANTHOCORIDAE)
}

\author{
K.C. Albernaz ${ }^{1 *}$, G.A. Carvalho², B.F.Carvalho², J.R. de Souza ${ }^{2}$ \\ ${ }^{1}$ Universidade deSão Paulo, Escola Superior de Agricultura “Luiz deQueiroz", Departamento de Entomologia, \\ Fitopatologia e Zoologia Agrícola, CP 9, CEP 13418-900, Piracicaba, SP, Brasil. E-mail: albernaz@esalq.usp.br
}

\section{RESUMO}

Predadores do gênero Orius alimentam-se de várias pragas de importância econômica, como tripes, moscas-brancas, afídeos, ácaros, ovos e pequenas lagartas de lepidópteros, contribuindo para sua regulação populacional. Objetivou-se avaliar a toxicidade tópica dos pesticidas (g i.a. 100 $\mathrm{L}^{-1}$ de água) mancozebe (160), clorotalonil (150), lufenurom (15), dicofol (36), bifentrina (2), triazofós $(40)$, metomil $(21,5)$ e acetamipride $(6)$, utilizados em crisântemo, sobre adultos de Orius insidiosus (Say). A aplicação foi realizada por meio de torre de Potter calibrada a $15 \mathrm{lb} / \mathrm{pol}^{2}$, com aplicação de $1,5 \pm 0,5 \mathrm{mg}$ de calda $/ \mathrm{cm}^{2}$ em adultos do predador com até $48 \mathrm{~h}$ de idade. A toxicidade dos produtos foi determinada pela porcentagem do efeito total $(\mathrm{E} \%)$, em função do número de insetos mortos até o oitavo dia após a aplicação dos compostos e da oviposição diária e total até os 15 dias após o período de pré-oviposição os quais foram classificados segundo critérios propostos pela International Organization for Biological Control (IOBC). Com base nos resultados, clorotalonil foi considerado inócuo ao predador (classe 1), enquanto mancozebe e dicofol foram levemente nocivos (classe 2), e lufenurom mostrou-se moderadamente nocivo (classe 3). Os demais produtos, bifentrina, triazofós, metomil e acetamipride foram considerados nocivos (classe 4) ao predador O. insidiosus. Em função da baixa toxicidade de clorotalonil, mancozebe e dicofol, esses compostos podem ser recomendados em programas de manejo visando a conservação dessa espécie de predador.

PALAVRAS-CHAVE: Cultivo protegido, pragas, Orius spp., pesticidas, seletividade.

\section{ABSTRACT}

TOXICITY OF PESTICIDES TO ADULTS OF ORIUS INSIDIOSUS (SAY, 1832) (HEMIPTERA: ANTHOCORIDAE). Predators of the genus Orius feed on many pests of economic importance, such as thrips, whiteflies, aphids, mites, eggs and small lepidopteran larvae, contributing to their population control. Thus, this work investigated the toxicity ( $\mathrm{g}$ a.i. $100 \mathrm{~L}^{-1}$ of water) of the pesticides mancozeb (160), chlorothalonil (150), lufenuron (15), dicofol (36), bifenthrin (2), triazophos (40), methomyl (21.5), and acetamiprid (6) used in chrysanthemum production on adults of the Orius insidiosus (Say) bug. The topical treatment consisted of using a Potter's tower adjusted to $15 \mathrm{lb} /$ $\mathrm{pol}^{2}$, with an application of $1.5 \pm 0.5 \mathrm{mg}$ of mixture $/ \mathrm{cm}^{2}$, on adults with age up to $48 \mathrm{~h}$. The toxicity of the pesticides for adults was determined by the percentage of the total effect (E\%), taking into account the daily mortality during 8 days after treatment with the pesticides, and daily and total oviposition during 15 days. Then, the pesticides were ranked according to criteria proposed by the International Organization for Biological Control (IOBC). Based on the results, chlorothalonil was considered harmless to the predator (class 1), while mancozeb and dicofol were classified as slightly harmful (class 2), and lufenuron was considered moderately harmful (class 3). The remaining products, bifenthrin, triazophos, methomyl, and acetamiprid were ranked as harmful (class 4 ) to the predator O. insidiosus. Therefore, due to the low toxicity of chlorothalonil, mancozeb and dicofol, these compounds can be recommended in management programs for the conservation of this species of predator.

KEY WORDS: Protected cultivation, pests, Orius spp., pesticides, selectivity.

${ }^{2}$ Universidade Federal de Lavras, Departamento de Entomologia, Lavras, MG, Brasil.

*Doutoranda ESALQ/USP, Departamento de Entomologia, Fitopatologia e Zoologia Agrícola. 


\section{INTRODUÇÃO}

O cultivo de crisântemo em ambiente protegido favorece a obtenção de produtos de alta qualidade, além da precocidade da cultura. Em contrapartida, também estão associados a esse método de cultivo o surgimento e a explosão populacional de pragas (OliveIrA, 1995; vAN LenTEREN, 2000), dentre as quais podem-se destacar os ácaros, afídeos, tripese moscasbrancas (BUENO, 2005). Estas pragas são responsáveis por sérios prejuízos aos agricultores, podendo comprometer a qualidade dos produtos e reduzir a produção (Oliveira, 1995). De acordo com Silveira et al. (2004), uma das alternativas para a manutenção de populações de alguns desses insetos-praga abaixo do nível de dano econômicoé o controlebiológico com a utilização do predador Orius insidiosus (Say, 1832) (Hemiptera: Anthocoridae).

A espécie $O$. insidiosus alimenta-se de presas em quase todos os estágios de desenvolvimento, possuindogrande capacidade de dispersão e de se esconder entre as folhagens e flores das plantas, podendo ocupar o hábitat de suas presas, destacando-se os tripes (BUENO, 2000).

Ressalta-se que o controle de tripes em cultivos protegidos de crisântemo vem sendo realizado principalmente por meio de produtos químicos, que geralmente são incompatíveis com a maioria dos agentes de controle biológico. Assim, o uso de compostos seletivos é uma importante estratégia para a conservação e manutenção de inimigos naturais em condições de cultivos protegidos. Desta forma, este trabalho teve como objetivo avaliar a toxicidade de pesticidas utilizados na cultura do crisântemo para o controle de pragas e doenças sobre o predador O. insidiosus.

\section{MATERIALEMÉTODOS}

Adultos do predador O. insidiosus foram obtidos da criação de manutenção do Laboratório deControle BiológicodeInsetos doDepartamentodeEntomologia da UFLA, conforme método de criação descrito por Mendes; Bueno (2001).

\section{Pesticidas avaliados}

Onome técnico, classe, dosagemeogrupoquímico de cada produto avaliado estão apresentados na Tabela 1. O tratamento controle foi feito apenas com água destilada.

As pulverizações dos pesticidas sobre os insetos foram realizadas por meio de torre de Potter regulada à pressão de $15 \mathrm{lb} / \mathrm{pol}^{2}$, com aplicação de $1,5 \pm 0,5 \mathrm{mg}$ de calda $/ \mathrm{cm}^{2}$, conforme as recomendações da
"International Organization for Biological and Integrated Control of Noxious Animals and Plants (IOBC), West Palaearctic Regional Section (WPRS)" (VeIre et al., 1996).

\section{Efeito dos pesticidas sobre adultos de O.insidiosus}

Para a realização deste bioensaio foram utilizados trinta e dois adultos (16 machos e 16 fêmeas) com até 48 horas de idade por tratamento. Os predadores foram colocados em placas de Petri de $15 \mathrm{~cm}$ de diâmetroe tratados topicamente por meio de pulverização dos produtos apresentados na Tabela 1. A cada pulverização, a torre de Potter foi lavada com água destilada e álcool.

Após as pulverizações, os insetos foram agrupados por casal e colocados em placas de Petri de $5 \mathrm{~cm}$ de diâmetro contendo ovos de Anagasta kuehniella (Zeller, 1879) (Lepidoptera: Pyralidae) oferecidos ad libitum como alimento a cada dois dias, e algodão umedecidoemágua destilada. Cada placa foi fechada com filme plástico de PVC e mantida em câmara climatizada regulada a $25 \pm 2^{\circ} \mathrm{C}$, UR de $70 \pm 10 \%$ e fotofase de 12 horas.

A mortalidade dos adultos foi avaliada diariamente após a aplicação dos produtos com auxílio de um microscópio estereoscópico (40x), sendo consideradomortooinseto quese manteveimóvel ao toquede um pincel.

Para os tratamentos que 24 horas após as pulverizações apresentaram fêmeas vivas, ofereceu-se uma haste de inflorescência de picão-preto Bidens pilosa L. sem o pólen com aproximadamente $4 \mathrm{~cm}$ de comprimento, envolvida na base por um chumaço de algodão umedecido com água destilada, que serviu como substrato de oviposição. As hastes foram oferecidas aos casais diariamente, por um período de 24 horas, durante 15 dias.

Para a verificação da viabilidade dos ovos, as hastes foram retiradas diariamente das placas e inseridas em pelets de ágar-água $1 \%$ e Nipagin $(0,112$ g100 $\mathrm{mL}^{-1}$ deágua) para maior tempo de conservação, eonúmero deovos/haste foi quantificado, sendo que cada haste foi armazenada em tubo de vidro de $8,5 \mathrm{~cm}$ de comprimento $\times 2,5 \mathrm{~cm}$ de diâmetro, fechado com filme plástico de PVC. Após sete dias, foi realizada a avaliação da viabilidade de cada ovo, sendo viáveis os que apresentaram opérculos abertos.

Odelineamento experimental utilizado foi deblocos casualizados, com nove tratamentos e quatro repetições, sendo cada parcela constituída por quatro casais de $O$. insidiosus.

Diariamente foi avaliada a mortalidade dos insetos até o oitavo dia após a aplicação dos produtos e o número diário e total de ovos depositados durante 15 dias, e a sua viabilidade. 
Tabela 1 - Nome técnico, classe, dosagem e grupo químico dos produtos fitossanitários avaliados.

\begin{tabular}{llcc}
\hline Produto técnico & Classe & $\begin{array}{c}\text { Dosagem } \\
\text { (g i.a. 100 L }{ }^{-1} \text { água) }\end{array}$ & Grupo químico \\
\hline mancozebe & fungicida & 160 & ditiocarbamato \\
clorotalonil & fungicida & 150 & isoftalonitrila \\
lufenurom & inseticida & 15 & benzoilfenilureia \\
dicofol & acaricida & 36 & prganoclorado \\
bifentrina & acaricida-inseticida & 2 & organofosforado \\
triazofós & acaricida-inseticida & 40 & carbamato \\
metomil & inseticida & 21,5 & neonicotinoide \\
acetamipride & inseticida & 6 & \\
\hline
\end{tabular}

\section{Análise estatística}

Os dados referentes à mortalidade diária de adultos até os oito dias após a aplicação dos pesticidas foram submetidos à análise de variância sem qualquer tipo de transformação, sendo que aqueles associados à mortalidade ao longo do tempo foram analisados por meio de regressão.

Os dados relativos aonúmero diário e total deovos em 15 dias foram transformados para $\sqrt{x+0,5}$ e submetidos à análise de variância. Para os dados de viabilidade dos ovos, realizou-se análise sem qualquer transformação.

As análises estatísticas foram realizadas por meio do programa proc GLM do SAS (SAS INSTITUTE, 1990). Nos casos em que o teste F da ANOVA foi significativo, a comparação de médias foi feita pelo teste de Tukey a 5\% de significância.

\section{Cálculos para classificação dos produtos avalia- dos conforme a IOBC}

Os dados de mortalidade foram corrigidos por meio da fórmula de Abbott (Аввотт, 1925): $\mathrm{Ma}=(\mathrm{Mt}$ -Mc) $/(100-$ Mc) $\times 100$, sendo que Ma = mortalidade corrigida em função do tratamento testemunha, $\mathrm{Mt}$ $=$ mortalidade observada no tratamento com o produto e $\mathrm{Mc}=$ mortalidade verificada no tratamento testemunha.

Para o cálculo do efeito total (E), utilizou-se a fórmula proposta por VAN DE VEIRE et al. (1996): $\mathrm{E}=$ $100 \%-(100 \%$ - Ma $) \times$ ER, sendo que Ma = mortalidade corrigida em função do tratamento testemunha e ER = Rt $/ \mathrm{Rc}$, onde ER = efeito do produto sobre o número de ovos colocados, $\mathrm{Rt}=$ número médio de ovos obtidos no tratamento inseticida e $\mathrm{Rc}=$ número médio de ovos obtidos no tratamento testemunha.

Após a determinação do efeito total, os produtos foram enquadrados em classes toxicológicas como: classe $1=$ inócuo $(\mathrm{E}<30 \%$ de mortalidade ou de redução da capacidade benéfica do predador), classe $2=$ levemente nocivo $(30 \% \leq \mathrm{E} \leq 80 \%)$, classe $3=$ moderadamente nocivo $(80<\mathrm{E} \leq 99 \%)$ e classe $4=$ nocivo $(E>99 \%)$, de acordo com a escala proposta por membros da IOBC (VAN De VeIRE et al., 1996).

\section{RESULTADOSEDISCUSSÃO}

Um dia após a aplicação dos produtos, lufenurom foi inócuo ao predador, com média de $3,1 \%$; mancozebe e clorotalonil causaram baixa mortalidade, com médias de 18,7 e 12,5\%, respectivamente; dicofol apresentou toxicidade intermediária, com média de 37,5\%; bifentrina, triazofós, metomil e acetamipride foram os mais prejudiciais aos adultos de $O$. insidiosus, causando mortalidades de 100, 100, 93,7 e 100\%, respectivamente (Fig. 1 e Tabela 2).

No decorrer das avaliações observou-se aumento significativo na mortalidade de O. insidiosus em todos os tratamentos, sendo que as médias de mortalidade apresentadas oito dias após a aplicação dos produtos foram de $31,2 \%$ para mancozebe; $34,4 \%$ para clorotalonil; $37,5 \%$ para lufenurom; $62,5 \%$ para dicofol e 96,8\% para metomil (Fig. 1). Resultados semelhantes aos do presente trabalho foram verificados por outros autores. Al-DeEB et al. (2001), avaliando o efeito de bifentrina (90 g i.a. ha ${ }^{-1}$ ) sobre adultos de $O$. insidiosus em casa-de-vegetação, verificaram que o produto foi tóxico ao predador, provocando alta mortalidade.

Os resultados obtidos com bifentrina neste estudo estão de acordo com a toxicidade de piretroides como fenpropatrina e deltametrina determinados por MoRAIS et al. (2003) e Torres et al. (2007), que encontraram $100 \%$ de mortalidade para O. insidiosus 24 h após serem tratados com esses produtos. 
Os resultados obtidos para acetamipride assemelham-se àqueles de NARANJO; AKEY (2004) que, avaliando a sua eficiência no controle da mosca-branca Bemisia tabaci (Gennadius, 1889) em condições de campo, constataram que esse produto reduziu significativamente a população de Orius tristicolor (White, 1879) e, também, aos de KilPatrick et al. (2005) que, ao estudarem a seletividade dos inseticidas neonicotinoides acetamipride, tiametoxam e imidaclopride, encontraram que todos causaram reduções em populações de O. insidiosus. A reduzida mortalidade provocada pelo lufenurom vem confirmar os resultados obtidos por Van de VeIRE et al. (2002), que constatou baixa toxicidade para adultos de Orius laevigatus (Fieber, 1860).

Quanto aos efeitos dos produtos testados na capacidade reprodutiva de fêmeas de O. insidiosus, observou-se que a oviposição diária foi reduzida por lufenurom ( 0,56 ovo) e dicofol ( 3,38 ovos); os demais produtos mostraram-se inócuos, com significativo aumento no número diário de ovos para o fungicida clorotalonil (9,23 ovos) quando comparado com a testemunha (5,4 ovos) (Tabela 3$)$. Uma hipótese para explicar esse fato é que tenha ocorrido efeito de hormoligose, segundo o qual agentes estressantes a um organismo numa determinada dosagem podem estimular o seu desempenho reprodutivo (LUCKEY, 1968). Entretanto, as verdadeiras razões para o aumento da fecundidade provocada por clorotalonil ainda não estão claras e necessitam de estudos mais aprofundados objetivando elucidar tal efeito.

Os produtos bifentrina, triazofós, metomil e acetamipride afetaram a sobrevivência dos percevejos (Tabela 2), o que impediu a avaliação dos parâmetros reprodutivos de O. insidiosus. Para o número total de ovos colocados em 15 dias (Tabela 3), verificou-se redução no tratamento à base de lufenurom, que permitiu oviposição de apenas 7,75 ovos, e um aumento significativo no número total de ovos para o fungicida clorotalonil, com média de 129,25 ovos, sendo observados 76,0 ovos para o tratamento testemunha. Os demais tratamentos não se diferiram da testemunha. Quanto à viabilidade dos ovos, observou-se que lufenurom reduziu esta característica biológica, com média de 9,2\%. Conforme constatado na Tabela 3, apesar de não terem sido detectadas diferenças significativas entre os demais tratamentos, dicofol apresentou baixa viabilidade, $46,5 \%$, enquanto os demais tratamentos apresentaram médias acima de $60 \%$.

Os resultados desta pesquisa referente ao lufenuron assemelham-se àqueles de EvANGELISTA et al. (2002), que obtiveram viabilidade dos ovos abaixo de $10 \%$ quando adultos do predador Podisus nigrispinus (Dallas, 1851) foram alimentados com

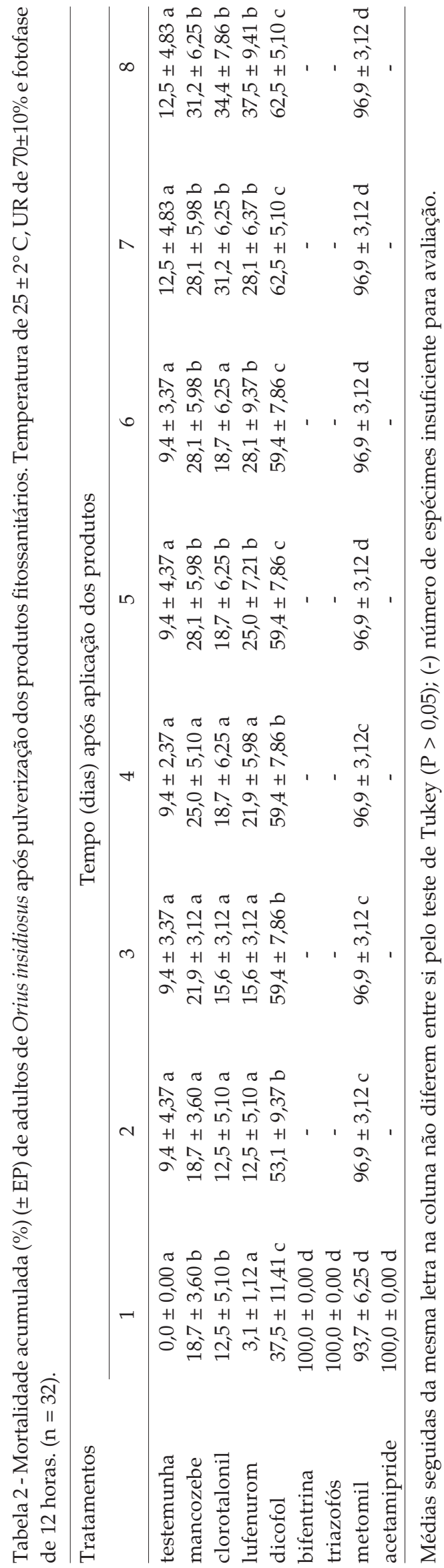

Arq. Inst. Biol., São Paulo, v.76, n.4, p.589-595, out./dez., 2009 
lagartas tratadas com lufenurom (50 g i.a. ha $\left.{ }^{-1}\right)$, e de VAN DE VEIRE et al. (1996), que constataram redução na oviposição de fêmeas de O. laevigatus tratadas com esse produto 10 gi.a. $L^{-1}$ deágua. A redução causada pelo lufenurom na oviposição e viabilidade de ovos podeser decorrente do efeito subletal desseinseticida, ou seja, aquele que não causa a morte dos insetos, porém afeta seu comportamento reprodutivo e/ou longevidade e capacidade de predação, entre outros aspectos (CROFT, 1990). Pode, ainda, ser devida à ação transovariana, o que foi constatado por ÁvILA; NAKANO (1999) em estudos desenvolvidos com Diabrotica speciosa (Germar, 1824), os quais verificaram efeito esterilizante do inseticida lufenurom a $0,03 \%$ sobre esta espécieapós ser alimentada com folhas defeijoeiro tratadas. Possivelmente, o produto ingerido pela fêmea pode ter sido transferido transovariamente para oembrião, afetando, dealguma forma, o seu desenvolvimento e impedindo a reprodução habitual desse inseto.

Verificou-sequeclorotalonil foienquadradonaclasse1(inócuo); mancozebeedicofolnaclasse2(levemente nocivos); lufenurom na classe 3 (moderadamente nocivo), ebifentrina, triazofós, metomileacetamiprideforam os mais prejudiciais ao predador $O$. insidiosus, sendo enquadrados na classe 4 (nocivos) (Tabela 4). Estes resultados são discrepantes dos obtidos por RocHA et al. (2006). Estes autores testaram a mistura de metalaxil + mancozebe em O. insidiosus, e esta foi considerada inócua(classe1). LeEetal.(1997) constataramalta toxicidade de mancozebe (75 WP) para ovos e adultos de Orius
sauteri(Poppius). As divergências deresultados podem estar relacionadas ao uso de metalaxil junto ao mancozebe, ou ainda decorrentes do uso de diferentes espécies do predador do gênero Orius bem como das metodologias utilizadas.

Em função da baixa toxicidade, o fungicida clorotalonil pode ser recomendado em programas de manejo de doenças em cultura de crisântemo sem que haja comprometimento para a espécie O. insidiosus. Para os demais produtos, recomendam-senovos testes em condições desemicampoecampo para a confirmação ou não de sua toxicidade a este predador.

Tabela 3 - Número diário e total de ovos de Orius insidiosus depositados durante 15 dias e sua viabilidade para predadores submetidos ou não a tratamento tópico com diferentes produtos. Temperatura de $25 \pm 2^{\circ} \mathrm{C}$, UR de $70 \pm 10 \%$ e fotofase de 12 horas.

\begin{tabular}{llcr}
\hline $\begin{array}{l}\text { Tratamentos } \\
\text { de ovos* }\end{array}$ & $\begin{array}{c}\text { No tiário } \\
\text { ovos } \\
\text { em 15 dias* }\end{array}$ & $\begin{array}{c}\text { Viabilidade } \\
(\%)^{*}\end{array}$ \\
\hline testemunha & $5,4 \pm 1,20 \mathrm{~b}$ & $76,0 \pm 18,53 \mathrm{~b}$ & $61,2 \pm 0,04 \mathrm{a}$ \\
mancozebe & $4,4 \pm 0,91 \mathrm{bc}$ & $61,5 \pm 27,52 \mathrm{~b}$ & $62,0 \pm 0,10 \mathrm{a}$ \\
clorotalonil & $9,2 \pm 2,12 \mathrm{a}$ & $129,2 \pm 31,91 \mathrm{a}$ & $68,4 \pm 0,08 \mathrm{a}$ \\
lufenurom & $0,6 \pm 0,11 \mathrm{~d}$ & $7,7 \pm 3,17 \mathrm{c}$ & $9,2 \pm 0,06 \mathrm{~b}$ \\
dicofol & $3,4 \pm 0,90 \mathrm{c}$ & $47,2 \pm 18,82 \mathrm{~b}$ & $46,5 \pm 0,16 \mathrm{a}$ \\
\hline
\end{tabular}

*Médias ( $\pm E P)$ seguidas da mesma letra na coluna não diferem entre si pelo teste de Tukey $(\mathrm{P}>0,05)$.

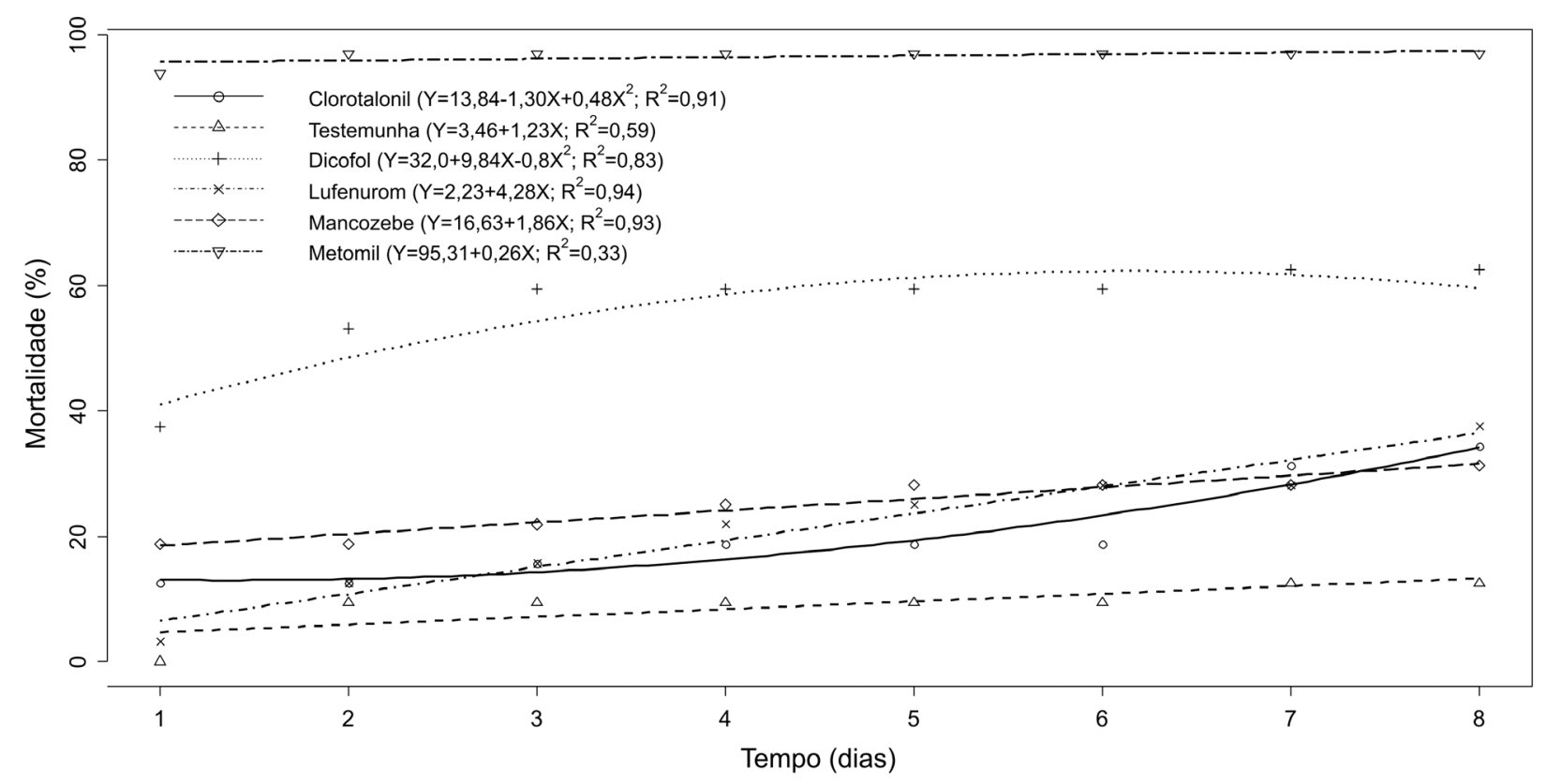

Fig. 1 - Curvas de mortalidade ao longo do período de avaliação para adultos de O. insidiosus tratados com os produtos fitossanitários. Temperatura de $25 \pm 2^{\circ} \mathrm{C}$, UR de $70 \pm 10 \%$ e fotofase de 12 horas. 
Tabela 4 - Mortalidade provocada pelos produtos a adultos de Orius insidiosus, efeito total (E) e classes de toxicidade dos produtos. Temperatura de $25 \pm 2^{\circ} \mathrm{C}$, UR de $70 \pm 10 \%$ e fotofase de 12 horas.

\begin{tabular}{|c|c|c|c|c|c|c|}
\hline Tratamentos & $\mathrm{n}^{1}$ & $\mathrm{M}(\%)^{2}$ & Mc $(\%)^{3}$ & $\begin{array}{c}\mathrm{N}^{\circ} \text { total de } \\
\text { ovos/15 dias }\end{array}$ & $\mathrm{E}(\%)^{4}$ & Classe $^{5}$ \\
\hline testemunha & 16 & 12,5 & - & 76,00 & - & - \\
\hline mancozebe & 16 & 31,25 & 21,42 & 61,50 & 37,14 & 2 \\
\hline clorotalonil & 16 & 34,38 & 25,00 & 129,25 & 0,00 & 1 \\
\hline lufenurom & 16 & 37,50 & 28,57 & 7,75 & 92,30 & 3 \\
\hline dicofol & 16 & 62,50 & 57,14 & 47,25 & 74,30 & 2 \\
\hline bifentrina & 16 & 100,00 & 100,00 & - & 100,00 & 4 \\
\hline triazofós & 16 & 100,00 & 100,00 & - & 100,00 & 4 \\
\hline metomil & 16 & 96,88 & 96,43 & - & 100,00 & 4 \\
\hline acetamipride & 16 & 100,00 & 100,00 & - & 100,00 & 4 \\
\hline
\end{tabular}

${ }^{1}$ Número de casais submetidos a cada tratamento.

${ }^{2}$ Mortalidade acumulada (\%) durante 8 dias após pulverização dos produtos sobre o predador.

${ }^{3}$ Mortalidade no tratamento corrigida pela fórmula de Abbott (Аввотт, 1925).

${ }^{4}$ Efeito total do produto sobre o predador.

${ }^{5}$ Classe de toxicidade segundo a IOBC.

\section{CONCLUSÕES}

O fungicida clorotalonil é inócuo ao predador $O$. insidiosus.

Mancozebe e dicofol são levemente nocivos aos adultos do predador.

Lufenuromémoderadamentenocivoao predador e bifentrina, triazofós, metomil e acetamipride são altamente tóxicos aos adultos de O. insidiosus.

Em função da baixa toxicidade de clorotalonil, mancozebe e dicofol, esses compostos podem ser recomendados em programas de manejo visando a conservação dessa espécie de predador.

\section{AGRADECIMENTO}

Ao Conselho Nacional de Desenvolvimento Científico e Tecnológico (CNPq), pela concessão da bolsa de estudos de mestrado para o primeiro autor.

\section{REFERÊNCIAS}

ABBOTT, W.S. A method of computing the effectiveness of an insecticide. Journal of Economic Entomology, v.18, p.265-267, 1925.

AL-DEEB, M.A.; WILDE, G.E.; KUN YAN ZHU. Effect of insecticides used in corn, sorghum, and alfalta on the predator Orius insidiosus (Hemiptera:

Anthocoridae). Journal of Economic Entomology, v.94, n.6, p.1353-1360, 2001.

ÁVILA, C.J.; NAKANO, O. Efeito do regulador de crescimento lufenuron na reprodução de Diabrotica speciosa (Germar) (Coleoptera: Chrysomelidae). Anais da Sociedade Entomológica do Brasil, v.28, n.2, p.293-299, 1999.

BUENO, V.H.P. Desenvolvimento e multiplicação de percevejos predadores do gênero Orius Wolff. In: BUENO, V.H.P. (Ed.). Controle biológico de pragas: produção massal e controle de qualidade. Lavras: UFLA, 2000. p.69-90.

BUENO, V.H.P. Controle biológico de tripes: pragas sérias em cultivos protegidos. Informe Agropecuário, v.26, n.225, p.31-39, 2005.

CROFT, B.A. Arthropod biological control agents and pesticides. New York: J. Wiley \& Sons, 1990. 723p.

EVANGELISTA JUNIOR, W.S.; SILVA-TORRES, C.S.A.; TORRES, J.B. Toxicidade de lufenuron para Podisus nigrispinus (Dallas) (Heteroptera: Pentatomidae). Neotropical Entomology, v.31, n.2, p.319-326, 2002.

KILPATRICK, A.L.; HAGERTY, A.M.; TURNIPSEED, S.G.; SULLIVAN, M.J.; BRIDGES, W.C. Activity of selected neonicotinoids and dicrotophos on nontarget arthropods in cotton: implications in insect management. Journal of Economic Entomology, v.38, n.3, p.814-820, 2005.

LEE, G.H.; CHOI, M.Y.; KIM, D.H. Effect of pesticides on predator Orius sauteri Poppius (Hemiptera: Anthocoridae). Journal of Crop Protection, v.39, p.61-66, 1997.

LUCKEY, T.D. Insect hormoligosis. Journal of Economic Entomology, v.61, n.1, p.7-12, 1968.

MENDES, S.; BUENO, V.H.P. Biologia de Orius insidiosus (Say) (Hemiptera: Anthocoridae) alimentado com Caliothrips phaseoli (Hood) (Thysanoptera: Thripidae). Neotropical Entomology, v.30, n.3, p.423-428, 2001. 
MORAIS, A. A.; CARVALHO, G.A.; MORAES, J.C.; GODOY, M.S.; COSME, L.V. Avaliação de seletividade de produtos fitossanitários utilizados na cultura do crisântemo a adultos de Orius insidiosus (Say, 1832) (Hemiptera: Anthocoridae) em laboratório. Ciência e Agrotecnologia, v.27, n.5, p.971-977, 2003.

NARANJO, S.E.; AKEY, D.H. Comparative efficacy and selectivity of acetamiprid for the management of Bemisia tabaci. Arizona Cotton Report, p.1-138, 2004.

OLIVEIRA, M.R.V. O emprego das casas de vegetação: vantagens e desvantagens. Pesquisa Agropecuária Brasileira, v.30, n.8, p.1049-1060, 1995.

ROCHA, L.C.D.; CARVALHO, G.A.; MOURA, A.P.; COSME, L.V.; VILELA, F.Z. Toxicidade de produtos fitossanitários utilizados na cultura do crisântemo para ovos e ninfas de Orius insidiosus (Say) (Hemiptera: Anthocoridae). Neotropical Entomology, v.35, n.1, p. 8392, 2006

SAS Institute. SAS/STAT Users guide. SAS Institute. Cary, NC, 1990.

SILVEIRA, L.P.C.; BUENO, V.H.P. van LENTEREN, J.C. Orius insidiosus as biological control agent of Thrips in greenhouse chrysanthemums in the tropics. Bulletin of Insectology, v.57, n.2, p.103-109, 2004.

TORRES, F.Z.V.; CARVALHO, G. A.; SOUZA, J. R.; ROCHA, C. L. Avaliação da toxicidade de inseticidas utilizados em roseira para adultos de Orius insidiosus (Say) (Hemiptera: Anthocoridae). Acta Scientiarum Agronomy, v.29, n.3, p.323-329, 2007.

van LENTEREN, J.C. A greenhouse without pesticides: fact or fantasy? Crop Protection, v.19, p.375-384, 2000.

van de VEIRE, M.; SMAGGHE, G.; DEGHEELE, D. A laboratory test method to evaluate the effect of 31 pesticides on the predatory bug, Orius laevigatus (Heteroptera: Anthocoridae). Entomophaga, v.41, n.2, p.235-243, 1996.

van de VEIRE, M.; STERK, G.; STAAIJ, M. van der; REMAKERS, P.M.J.; TIRRY, L. Sequential testing scheme for the assessment of the side-effects of plant protection products on the predatory bug Orius laevigatus. Biocontrol, v.47, p.101-113, 2002.

Recebido em $7 / 10 / 08$

Aceito em 9/10/09 